NBER WORKING PAPERS SERIES

A FALLACY OF COMPOSITION

Ricardo J. Caballero

Working Paper No. 3735

NATIONAL BUREAU OF ECONOMIC RESEARCH

1050 Massachusetts Avenue

Cambridge, MA 02138

June 1991

I am grateful to Phillip Cagan, Andrew Caplin, Mohammad Hammour, Anil Kashyap and Brendan O'Flaherty for very useful comments. This paper is part of NBER's research program in Economic Fluctuations. Any opinions expressed are those of the author and not those of the National Bureau of Economic Research. 
NBER Working Paper \#3735

June 1991

\section{A FALLACY OF COMPOSITION}

\section{ABSTRACT}

The representative agent framework has endowed macroeconomists with powerful microeconomic tools. Unfortunately, it has also blurred the distinction between statements that are valid at the individual level from those that apply to the aggregate. In this paper I argue that probability theory puts strong restrictions on the joint behavior of a large number of units that are less than fully synchronized, and that many fallacies arise from disregarding these restrictions. For example, the observation that the aggregate price level is more rigid to downward changes than to upward changes, has led many authors to suggest asymmetries at the firm level as responsible for the alleged macroeconomic fact. However, the basic insight developed in this paper shows that asymetric pricing policies at the firm level do not necessarily imply asymetries in upward and downward adjustments of the aggregate price level; and asymetries in the aggregate price level need not come from asymmetries at the firm level. Similarly, asymmetric factor adjustment costs at the firm level need not imply asymetric responses of the aggregate capital stock and the level of employment to positive and negative shocks.

Fallacy of composition: A fallacy in which what is true of a part is, on that account alone, alleged to be also true on the whole. Samuelson (1955)

Ricardo J. Caballero Department of Economics Columbia University New York, NY 10027 NBER and 
The representative agent framework is one of the most important tools for macroeconomists. On the one hand, sophisticated optimization arguments can be directly applied to aggregate data. On the other, microeconomic arguments are typically "intuitive" and therefore easier to handte; it is not rare for each new macroeconomic fact raised, to hear several appealing microeconomic stories accounting for it.

Of course, researchers have never pretended that all agents are literally the same in every dimension, but only that idiosyncrasies have no chief impact on the aggregate. This argument is certainly valid in many cases, however it is not universally true. In some important cases, idiosyncrasies not only do not wash away but also undo - at least partially - the basic microeconomic feature used to provide the macroeconomic argument. Perhaps the most prominent and extreme example of this is due to Caplin and Spulber (1987), where menu cost pricing at the firm level is fully consistent with complete aggregate price flexibility.

In this paper I attempt to isolate the mechanism underlying the source of several fallacies of composition. I argue, through a simple example, ${ }^{1}$ that the essence of these fallacies relies on the fact that direct microeconomic arguments do not consider the strong restrictions that probability theory puts on the joint behavior of a large number of units that are less than fully synchronized; put differently, the endogenous evolution of the cross section distribution of individual units is often restricted, by the microeconomic problem itself, in such a way as to rule out many direct microeconomic explanations of aggregate phenomena.

Examples of such fallacies are plenty, both in the literature and in everyday discussions. For example, the observation that the aggregate price level is more rigid to downward changes than to upward changes, has led many authors to suggest asymmetries at the firm level as responsible for the alleged macroeconomic fact. ${ }^{2}$ However, the basic insight developed in this paper shows that asymmetric pricing policies at the firm level do not necessarily imply asymmetries in upward and downward adjustments of the aggregate price level; and asymmetries in the aggregate price level need not come from asymmetries at the firm level. Similarly, asymmetric factor adjustment

\footnotetext{
'Caballeto and Engel (1989a,b) and (1990) develop the basic methodology. to analyze economies in which transection costo yield a prevalent role for idiosyncrasies. In this context, the dynamic behavior of aggregate variables depends in nontrivial ways on the evolution of the crose mection distribution.

${ }^{2}$ For an accounting of aggregate price level asymmetries, see Cagan (1979); for an allempl to use direct microeconomic arguments to account for such asymmetry, see Kuran (1983). For evidence on the abeence of price aymmetry at the firm level, see Carlton (1986).
} 
costs at the firm level need not imply asymmetric responses of the aggregate capital stock and the level of employment to positive and negative shocks.

To illustrate these issues I use as an example the recent finding by Davis and Haltiwanger (1989) and Blanchard and Diamond (1989) (the DH/BD fact, hereafter), that the cyclic beliavior of gross job reallocation in the U.S. is driven by job destruction as opposed to job creation. Our natural tendency is to propose microeconomic asymmetries (e.g. bankruptcy, asymmetric adjustment costs, etc.) as an explanation for this. I show below, however, that direct arguments based on microeconomic job creation and destruction asymmetries do not necessarily imply aggregate flow asymmetries. Of course the arguments used extend to the aggregate price level asymmetry and capital stock examples mentioned above as well as many others.

The remainder of the paper is organized in four sections and several appendices. The basic microeconomic model together with the corresponding limit probability arguments are presented in Section 1. The long run behavior of aggregate flows is illustrated in Section 2, and dynamics are discussed in Section 3. Conclusions are given in Section 4, and the appendices present some of the derivations and additional empirical properties of the models discussed in the paper.

\section{Microeconomic Behavior}

The model presented here is highly stylized and is devoid of the microeconomic theory justifying the behavior of individual units. Furthermore, the policy rules adopted by these units are assumed to be invariant to the different experiments performed. In most circumstances the latter is unrealistic and suboptimal but it permits us to isolate the aggregation issues more cleanly; no important qualitative conclusion depends on this. ${ }^{3}$

Let each individual firm $i$ have a desired (frictionless) level of employment at time $t$, equal to $L_{i t}^{*}$. Due to some friction (e.g. hiring and firing costs), actual employment at the firm level $\left(L_{i t}\right)$ is not always equal to the frictionaless optimal level; the difference between $L_{i t}$ and $L_{i t}^{*}$ is denoted by $D_{i t}$. Suppose now that firms have a threshold rule - as is typically the case in the presence of non-convexities in the adjustment technology (see Bertola and Caballero 1990) - so they fire workers (destroy jobs) when $D_{i t}$ crosses an upper threshold $T$ and hire (create jobs)

\footnotetext{
${ }^{3}$ See Bertola and Cabaliero (1990) for a justification of the microeconomic rules adopted here, and a discussion of the dependence of these rules on deep parameters.
} 
when $D_{\text {it }}$ crosses a lower threshold $B$. The quit rate is zero, so actual employment is constant at all other instances.

The frictionless level of employment is driven by a simple random walk:

$$
L_{i t}^{*}=L_{i t-1}^{*}+ \begin{cases}1 & \text { with probability } 1 / 2 \\ -1 & \text { with probability } 1 / 2\end{cases}
$$

The asymmetry takes the following form: during a job creation period the firm hires (II) less workers than it dismisses $(F)$ during job destruction periods (as would happen, for example, when larger fixed costs are involved in job destruction than in job creation). To fix ideas, let me start with a very simple case in which $T=1, B=-1, H=1$ and $F=2$.

In this case $D_{i \ell}$ can take only three values: $-1,0$ and 1 . When $D_{i t}$ is at -1 , there is a probability equal to $1 / 2$ that desired employment will increase further, leading the firm to hire another worker. In this case $D_{i t+1}$ remains at the same position as $D_{i l}$ since the hiring offsets the shock $\left(D_{i \ell+1}=-1-1+1=-1\right)$. It is equally likely that desired employment falls, so that the firm does not act and $D_{i t+1}=0$. When $D_{i t}=0$, there is neither hiring nor firing at $t+1$, and $D_{i t+1}$ can be either at -1 or 1 with equal probability. Finally, when $D_{i t}=1$ there is a probability $1 / 2$ that in the next period the firm will destroy two jobs, and $D_{i t+1}=0$ with probability 1.

Let $p_{t}(-1), p_{t}(0)$ and $p_{t}(1)$ be the probability of $D_{i \ell}$ being at $-1,0$ and 1 , respectively (of course they sum up to one at every time $t$ ). For now I concentrate on the characteristics of the limiting probability vector, denoted by $p{ }^{4}$

By definition, each stationary probability $p(h)$ must be equal to the sum of the probabilities $p(k)$ weighted by the probabilities of moving from each state $k$ to state $h$. Thus,

$$
\begin{gathered}
p(0)=\frac{1}{2} p(-1)+p(1), \\
p(-1)=\frac{1}{2} p(0)+\frac{1}{2} p(-1), \\
p(1)=\frac{1}{2} p(0),
\end{gathered}
$$

and of course the adding up condition must be satisficd:

$$
p(-1)+p(0)+p(1)=1 .
$$

\footnotetext{
'It is immediate to show that this Markov chain is ergodic; i.e. starting from any vector of probsbilities po, eventually the time subindex becomes irrelevant.
} 
Stationary probabilities caul be interpreled as the relative time spent in each state. A direct consequence of this is that job creation will occur with frequency $(1 / 2) p(-1)$ and job destruction with frequency $(1 / 2) p(1)$. But

$$
p(1)=\frac{1}{2} p(-1),
$$

thus the counterpart of the asymmetry in the size of hiring and firing, is that job creation occurs twice as often as job destruction. This very simple observation is at the root of the results obtained for the aggregate level in the next section, where probability statements for individual firms become cross section statements.

\section{Aggregate Long Run Counterbalance Effect}

Suppose now that there is a large number of firms following identical hiring and firing rules with their respective $L_{i t}^{*}$ paths described by standard random walks.

Also assume, for the time being, that firms have the same initial $D_{i t}$ and that their shocks are perfectly correlated. In this case, all the statements for the individual firm are valid for the aggregate. Most importantly, job creation occurs twice as often as job destruction but when they occur, job destruction is twice as large as job creation; the strong microeconomic asymmetry remains intact at the aggregatelevel validating the microeconomic story of aggregate phenomena. This, however, is an extreme version of the representative agent model, where at all times everything is exactly the same for every firm.

In this paper, I keep the assumption of identical policies but allow idiosyncrasies to be reflected in a less than perfect correlation of shocks across firms. It is convenient to start with the case in which this correlation is zero. In this case, the Glivenko-Cantelli theorem (see Billingsley 1986) allows us to interpret statements about probabilities at the firm level, as statements about the fraction of firms in different positions of the state space of $D_{i t}$. In other words, starting from any cross section distribution on the space of the $D_{i i}$ 's, eventually the cross section becomes stationary; i.e. individual firms change their positions continuously but the cross section distribution remains unchanged.

The importance of this is that eventually job destruction and job creation become constant. In this state there is a fraction $(1 / 2) p(-1)$ (per period) of firms hiring one worker each, and a fraction $(1 / 2) p(1)$ of firms firing two workers each. But as $p(1)=(1 / 2) p(-1)$, job creation and 
destruction are not only constant but equal. Thus, from the point of view of the flow variables, the microeconomic asymmetry washes away completely.

In what follows, I reduce the impact of the state space discreetness by enlarging it, so the $D_{i t}$ 's take values between -7 and 7 (i.e. there are 15 positions in the state space), and the asymmetry is made even more pronounced: job creation is still equal to one, whereas job destruction is now equal to six. ${ }^{5}$ So, on the one hand, firms wait until the shortage of workers (respect to the frictionless optimal) exceeds (in absolute value) -7 to hire one worker, returning to the position $D_{i \ell}=-7$; on the other, they wait until the excess of labor go beyond 7 to fire six workers, returning to the position $D_{i t}=-2$. Of course, the basic fact that flow asymmetries wash away is invariant to any of these modifications. Figure 1 illustrates the invariant cross section distribution. The asymmetry in the size of hiring and firing adjustments is perfectly offset by the distribution of firms in state space; $p(-7) / p(7)$ is equal to $6 / 1$ (the inverse of the ratio of the adjustment sizes), hence $(1 / 2) p(-7)=6(1 / 2) p(7)$ and job creation is equal to job destruction.

In the absence of aggregate fluctuations, the size of the flows in and out of employment has nothing to do with the microeconomic asymmetry.

\section{Aggregate Dynamic Counterbalance Effect}

When aggregate fluctuations are added, the cross section does not reach an invariant state; therefore, job creation and destruction are no longer constant. However, the statements of the previous section are true for the average flows: average job destruction is equal to average job creation, regardless of the degree of microeconomic asymmetry.

On average, since the ergodic probability distribution of each firm is still as in Figure 1, there is six times as many firms near the job creation barrier than near the job destruction barrier. More importantly, I provide examples below that show that provided that idiosyncrasies are present, ${ }^{6}$ the average cross section distribution effect impedes the microeconomic asymmetry from generating similar asymmetry in the aggregate dynamic flows.

\footnotetext{
${ }^{3}$ Nole that the tact that the center of the state gpace is kept at rero is junt a matler of notational convenience; a change in the center is eacily obtained by adding a constant to the definition of the $D_{i 1}$ 's.

-Otherwise the impact of initial conditions in general does not wash away.
} 


\subsection{Aggregate cycles}

Before introducing aggregate uncertainty, it is convenient to study the implications of adding aggregate deterministic cycles to the model discussed above. This framework puts the strictest tests to the proposition above, as it strengthens the chances for the microeconomic asymmetry to permeate the aggregate (this is discussed in more detail below).

To consider aggregate cycles, I modify the model above by changing the microeconomic probabilities of positive and negative shocks. The $L_{i t}^{*}$ are now driven by switching random walks. In "good" times:

$$
L_{i t}^{*}=L_{i t-1}^{*}+ \begin{cases}1 & \text { with probability } \lambda_{g} \\ -1 & \text { with probability } 1-\lambda_{g}\end{cases}
$$

and in bad times

$$
L_{i t}^{*}=L_{i t-1}^{*}+ \begin{cases}1 & \text { with probability } \lambda_{b} \\ -1 & \text { with probability } 1-\lambda_{b},\end{cases}
$$

with $1 / 2<\lambda_{g}<1$ and $0<\lambda_{b}<1 / 2$. In addition to transition probabilities at the individual level, if shocks are independent (given $\lambda_{g}$ ) across firms, ${ }^{7} \lambda_{g}$ represents the fraction of firms that receive a good shock in good times, whereas $\lambda_{b}$ is the fraction of firms that receive a good shock during bad times; good and bad times are then determined by whether more or less than half of the firms are affected by good shocks, respectively. This classification of states of the overall economic environment is arbitrary but hardly controversial.

A full cycle is defined as a given string of consecutive good periods followed by a given string of bad periods. Since in this case the transition matrix -i.e. the matrix that determines the fraction of firms that, being at each position $h$, move to positions $k$ in the state space- is not constant, there is no longer a stationary cross section distribution; however it is fairly easy to show that there is a stationary cycle of cross section distributions. The simplest case to illustrate this is one in which good and bad periods alternate; in this example, the transition matrices during good and bad times are denoted by $P_{g}$ and $P_{b}$, respectively (their explicit form is not important). The products $P_{g} P_{b}$ and $P_{b} P_{g}$ are denoted by $P_{g b}$ and $P_{b g}$, respectively. Assume now that the first period (time 1) is good; then the Markov chain can be decomposed into two

\footnotetext{
'Or at lesst satisfy some mixing condition.
} 
chains, one for even periods and one for odd periods:

$$
p_{t}=p_{t-2} P_{g b} \quad \text { if } t \text { is even, }
$$

and

$$
p_{t}=p_{t-2} P_{b d} \quad \text { if } t \text { is odd. }
$$

Again, it is trivial to show that both chains are ergodic, thus eventually the time subindices can be removed, obtaining two stable but alternating cross sections, $p_{g b}$ and $p_{b g}$.

This can be generalized fairly easily as long as there is some repeated pattern in the way good and bad times arrive. ${ }^{8}$ In what follows I continue assuming that there are 15 positions in the state space, that firms hire one worker when creating jobs and dismiss six worker when destructing jobs. In addition $I$ assume that $n_{g}$ and $n_{b}$, the number of consecutive good and bad periods, respectively, are equal to five. Certainly $n_{g}$ and $n_{b}$ need not be equal; $;$ I make this assumption to isolate the main issue addressed in this paper from aggregate sources of asymmetry. For the same reason, I assume $\lambda_{6}=1-\lambda_{g}$. Later, I relax all these assumptions.

Recalling that aggregate job creation and desiruction flows are equal to $\lambda_{t} p_{t}(-7)$ and $6(1-$ $\left.\lambda_{l}\right) p_{i}(7)$, respectively, and that $\lambda_{g}=1-\lambda_{b}$, any strong asymmetry in these flows dynamics must be reflected in a strong asymmetry in the evolution of the fraction of firms near each of the boundaries. Furthermore, given that firing, when occurs, is six times larger than hiring, when occurs, it seems sensible to look at whether there is any evidence of larger volatility in $6 p_{l}(7)$ than in $p_{t}(-7)$ to settle the issue. Figures $2 \mathrm{a}$ and $2 \mathrm{~b}$ show that such asymmetry is not present in the example provided here. Figure 2a illustrates the path of $p_{t}(-7)$ for a complete cycle, and Figure $2 \mathrm{~b}$ does the same for $6 p_{t}(7)$. Even though they are not mirror images of each other, $6 p_{t}(7)$ is far from having peek to trough differences six times larger than that of $p_{t}(-7)$. These figures correspond to the case $\lambda_{g}=0.65$, but the fundamental result is invariant to reasonable values of this parameter.

The story of the previous figures is not complete since the covariance between $p_{l}(-7)$ and $\lambda_{t}$ may differ from that of $p_{t}(7)$ and $\left(1-\lambda_{t}\right)$; the figures below show that this is not the case, however. Figure $3 a$ shows the path of aggregate job creation (solid line) and job destruction

\footnotetext{
'The lack of such pattern is what determines that when the allocation of good and bad times is olochastic, there is no form of stable cross section distribution. See the next subsection.

'Lel alone equal to frve!
} 
(dashed lines) for four complete cycles. It is apparent that, even though at the microeconomic level job destruction (when it occurs) is six times larger than job creation (when it occurs), no comparable implication carries over to the aggregate flows (the peak to trough differences in both series is about the same). Figure $3 \mathrm{~b}$ reproduces Figure $3 \mathrm{a}$ but with $n_{g}=n_{b}=30$; the basic conclusion is the same.

It is interesting to note, however, that microeconomic asymmetries do have implications for aggregate dynamics (job destruction and creation cycles are not identical) but these are far from trivial and direct. Comparing figures $3 a$ and $3 b$ it is possible to see that fluctuations in aggregate job destruction flows may be smaller or larger than fluctuations on aggregate job creation flows, depending upon the path of aggregate shocks. In this particular case, the asymmetry in the direction of the microeconomic asymmetry increases as both "states of the world" become more persistent; the reason for this is that a long string of positive shocks bunch more units together than a long string of bad shocks, since the return point is farther from the trigger point in the job destruction case than in the job creation case. Thus, when a long string of positive shocks is followed by some negative shocks the units are highly bunched creating an "over-response" of job destruction. More important than the explanation per-se (for the purpose of this paper), is the fact that the role of microeconomic asymmetries on aggregate fluctuations is far more intricate than suggested by direct microeconomic stories.

To summarize, when aggregate fluctuations are present, the steady state result of the previous section is preserved in a much more subtle way; the offsetting of the microeconomic asym. metry through the cross section distribution occurs at the mirror image states of the world (very good booms with very bad recessions, mild booms with mild recessions, etc.). The consequence of this is that fluctuations in aggregate job creation and destruction are not fundamentally different in their size, in spite of the strong microeconomic asymmetry.

\subsection{Aggregate uncertainty}

The next step is to show that the fallacy of composition is not an artifact of the particular form of the deterministic cycle considered above. The same conclusions carry over to the case in which changes in the state of the world (the $\lambda_{g}$ ) are stochastic and there is no stationary cross 
section distribution. ${ }^{10}$

Assume that each period is good with probability $1 / 2$ and bad with the same probability. It is easy to show"t that in this case:

$$
\lambda_{g}=\frac{1}{2}(1+\delta),
$$

where $\delta$ is the fraction of the total uncertainty faced by an individual firm that is due to aggregate uncertainty. ${ }^{12}$ A value of $\lambda_{g}$ of 0.65 -as used here-corresponds to a $\delta$ equal to 30 percent; i.e. 30 percent of the total uncertainty faced by individual firms comes from aggregate uncertainty. In good times $65 \%$ percent of the firms receive a positive shock and $35 \%$ a negative one. During bad times these proportions are reversed. ${ }^{13}$ Again, none of the main conclusions of the paper depends on the precise value of $\lambda_{g}$ assumed.

Table 1 provides Monte Carlo evidence on the claim that microeconomic asymmetries do not necessarily imply aggregate asymmetries. Row 1 (Asymm.) corresponds to the resuits obtained from 1,000 replications of samples of 200 hundred periods (the parameters are those described above). ${ }^{14}$ Row 2 (Symm.) corresponds to the same experiment (with the same seeds for the random generator) but for a case in which there is no microeconomic asymmetry (firms hire and fire one worker). The first two columns are the mean job creation $\left(\bar{x}_{j o b c}\right)$ and destruction $\left(\bar{x}_{\text {jobd }}\right)$, respectively (standard deviations in parenthesis). There is no evidence of significant difference between average job creation and job destruction in any of the two cases, nor is there significant difference across the two cases. Both statements are a refiection of the long run aggregate counterbalanoe mechanism. Columns 3 and 4 report the average standard deviations of aggregate job creation $\left(\sigma_{\text {jobc }}\right)$ and job destruction $\left(\sigma_{\text {jobd }}\right)$ (standard deviations in parenthesis); they show that microeconomic asymmetries neither add to the overall aggregate flow fluctuations nor, more importantly, generate significant differences in the fluctuations of job creation and job

\footnotetext{
${ }^{10}$ Not even in the weaker sense described in the previous subsection.

"See the appendix.

${ }^{17}$ Note that $\lambda_{6}=\frac{1}{2}(1-\delta)$.

${ }^{15} A$ nice property of the model here described is that in the continuous time limit the stochastic process describing the path of each $L_{i}^{*}$ converges to the sum of two independent Brownian motion, one for the common shock across firms and another for the idionyncratic component.

"Strictly speaking, using substantially longer sample sizes take the simulations closer to their theoretical limits; I have chosen relatively amall samples in order to andyze the resule in a context more comparable to that of the actual empirical evidence. Conversely, smaller samples increase the dispersion of the results.
} 
Table 1: Aggregate Flows Statistics

\begin{tabular}{|c|c|c|c|c|c|}
\hline & $\bar{x}_{j o b c}$ & $\bar{x}_{\text {jobd }}$ & $\sigma_{j o b c}$ & $\sigma_{\text {jobd }}$ & $\rho_{\sigma_{j o b c}, \sigma_{j o b d}}$ \\
\hline Asymm. & $\begin{array}{c}0.0435 \\
(0.0106)\end{array}$ & $\begin{array}{c}0.0437 \\
(0.0106)\end{array}$ & $\begin{array}{c}0.0299 \\
(0.0069)\end{array}$ & $\begin{array}{c}0.0300 \\
(0.0068)\end{array}$ & -0.42 \\
\hline Symm. & $\begin{array}{c}0.0362 \\
(0.0103) \\
\end{array}$ & $\begin{array}{c}0.0364 \\
(0.0100) \\
\end{array}$ & $\begin{array}{c}0.0250 \\
(0.0068) \\
\end{array}$ & $\begin{array}{c}0.0250 \\
(0.0064) \\
\end{array}$ & -0.50 \\
\hline
\end{tabular}

destruction (the ratio of job destruction standard deviation to job creation standard deviation is above 2.5 at the firm level). This is a reflection of the dynamic counterbalance mechanism.

The last column of Table 1 shows the correlation between the standard deviation measures. In both cases - the asymmetric and symmetric - the correlation is highly negative; this means that within any given sample there can be important differences between the standard deviations of aggregate job creation and job destruction. This is illustrated in Figures $4 \mathrm{a}$ and $4 \mathrm{~b}$. They present the histograms of the log difference of job creation and job destruction standard deviations, for the asymmetric and symmetric case, respectively, resulting from the Monte Carlo experiment described above: Both figures show that samples in which the standard deviation of one of the flows is fifty percent larger than that of the other one are not too rare. What is more important, however, is that there is no significant differences across the figures nor a systematic bias in the direction of larger standard deviations of job destruction in Figure 4a.

The results in Table 1 and Figures $4 \mathrm{a}$ and $4 \mathrm{~b}$ also show that large aggregate asymmetries may arise in certain samples. These are not, however, a consequence of the microeconomic asymmetry (compare Figures $4 \mathrm{a}$ and $4 \mathrm{~b}$ ), but of the particular draw of good and bad aggregate realizations. This suggests that if we are to provide meaningful explanations for phenomena where heterogeneity is non trivial much more effort is needed in modeling the evolution of cross section distributions and their driving forces.

The following is a simple example of this type of explanations, where the samples generated are characterized by aggregate job destruction series that are typically more volatile than job creation series. For this let me modify the previous example so expansions are typically longer 
lived than contractions; I capture this by letting $q$, tbe probability of a good aggregate real. ization, be larger than 0.5 . In particular, I choose 0.75 , by which a positive aggregate shock is three times more likely than a negative one. In order to maintain the average equality between job creation and job destruction, ${ }^{15}$ I compensate the increase in $q$ with a deterioration of bad times (i.e. $\lambda_{b}<1-\lambda_{g}$ ). ${ }^{16}$ It is easy to check that, in order for the aggregate flows to be equal on average, it must be the case tliat:

$$
\lambda_{b}=\frac{1}{2}\left[1+\left(1-2 \lambda_{g}\right) \frac{q}{1-q}\right] .
$$

And tbis implies that equation (1) is replaced by:

$$
\lambda_{g}=\frac{1}{2}\left(1+\delta \sqrt{\frac{1-q}{q}}\right)
$$

and

$$
\lambda_{b}=\frac{1}{2}\left(1-\delta \sqrt{\frac{q}{1-q}}\right) .
$$

It is also interesting to notice that in this case tbe contribution of idiosyncratic shocks to the uncertainty faced by firms changes with the state of the world; in bad times more units move together. This can be conveniently summarized by the ratio of conditional (on whether the state is good or bad, respectively) variances (minus one), $I$ :

$$
I \equiv \frac{\lambda_{g}\left(1-\lambda_{g}\right)}{\lambda_{b}\left(1-\lambda_{b}\right)}-1 .
$$

This index is equal to zero when $\lambda_{g}=1-\lambda_{b}$ and positive when $0.5<\lambda_{g}<1-\lambda_{b}$, as is the case here. In this model, therefore, negative aggregate shocks are accompanied by a decrease in the relative importance of reallocation shocks. With a slight abuse of terminology, I call this change in relative importance of both shocks, a change in the "intensity" of reallocation shocks (see DH).

Table 2, under these new assumptions, is the equivalent to Table 1 . Columns 3 and 4 show that, on average, job destruction is more volatile than job creation, regardless of the microeconomic asymmetry. Also notice that the volatility of job destruction varies substantially across samples (see the standard deviations in parentbesis). Figures $5 \mathrm{a}$ and $5 \mathrm{~b}$ below reveal this

\footnotetext{
15J.e. to avoid adding a drift to employment that would clutter the comparisons with the previous section

${ }^{10}$ Nole that many models will provide agymetric aggregate flows under these assumptions.
} 
Table 2: Aggregate Flows Statistics

\begin{tabular}{||c|c|c||c|c||c||}
\hline \hline & $\bar{x}_{\text {jobc }}$ & $\bar{x}_{\text {jobd }}$ & $\sigma_{\text {jobc }}$ & $\sigma_{\text {jobd }}$ & $\rho_{\sigma_{\text {oboc }}, \sigma_{\text {jobd }}}$ \\
\hline \hline Asymm. & $\begin{array}{c}0.0508 \\
(0.0198)\end{array}$ & $\begin{array}{c}0.0506 \\
(0.0227)\end{array}$ & $\begin{array}{c}0.0492 \\
(0.0141)\end{array}$ & $\begin{array}{c}0.0710 \\
(0.0306)\end{array}$ & -0.47 \\
\hline Symm. & $\begin{array}{c}0.0423 \\
(0.0195)\end{array}$ & $\begin{array}{c}0.0420 \\
(0.0214)\end{array}$ & $\begin{array}{c}0.0416 \\
(0.0149)\end{array}$ & $\begin{array}{c}0.0588 \\
(0.0286)\end{array}$ & -0.53 \\
& \multicolumn{6}{|c|}{$\delta=0.3, g=0.75, \lambda_{g}=0.65, \lambda_{b}=0.05}$. \\
\hline
\end{tabular}

more clearly; it is not rare to find the log-difference of the standard deviations of job creation and job destruction to be lower than -1 .

The mechanism through which this happens is, again, firmly rooted in simple probability laws. Typically firms here receive positive shocks; these tend to bunch them near the hiring barrier (even in the symmetric case), although not completely since in good times idiosyncratic shocks are important $\left(\lambda_{g}<<1\right)$. When bad times come, firms find themselves relatively bunched and have no time to spread out since bad times are accompanied by smaller idiosyncrasies $\left(\left(1-\lambda_{b}\right) \approx 1\right)$; the result is abrupt aggregate job destruction as the cluttered firms hit their firing barriers. Conversely, when good times come after bad times, idiosyncrasies reappear, thus firms typically do not reach their hiring barrier simultaneously.

This mechanism is better isolated in the context of the model with deterministic aggregate cycles; for this I let $n g=45$ and $n b=15$ (thus, $\left(n_{g} / n_{b}\right)=(q /(1-q))$ ). Figure 6 a depicts four complete cycles for aggregate flows (it should be compared with figures $3 a$ and $3 b$ ), illustrating the asymmetry in job creation and job destruction dynamics, and figure $6 \mathrm{~b}$ illustrates the path of total employment.

\section{Conclusion}

The complexities underlying many of the issues I have discussed in this paper can be significant, however, the basic message is not: direct application of microeconomic explanations to aggregate data can be seriously misleading, since they typically do not lake into account the natural probability forces that tend to undo such explanations. 
The principle is far more general than the simple examples used in this paper. For example, it would certainly apply to circumstances in which firms adjust their factors of production and/or prices at different speeds in the upward and downward directions (e.g. as would happen in an asymmetric convex cost of adjustment model). In this example (assuming no drift) firms would typically spend more time in the slow region, thus the cross section would tend to have a larger fraction of firms in this region; as a consequence, the aggregate would exhibit substantially less asymmetry in its instantaneous response to positive and negative shocks than individual firms would.

This paper should not be interpreted as an argument for the irrelevance of microeconomic stories as explanations of aggregate phenomena. ${ }^{17}$ Underlying any cross section story there has to be a microeconomic story. Furthermore, even though long episodes of relatively stable stochastic environments disperse firms on the state space in such a way that certain aspects of microeconomic arguments become second order most of the time, they are not necessarily so at all times; very large events reshape the cross section and reduce the relevance of ergodic arguments, allowing microeconomic stories to permeate the aggregate more freely.

The point emphasized, however, is that we need to be substantially more careful when using direct microeconomic arguments to account for aggregate phenomen $\mathrm{a}^{18}$.

\footnotetext{
${ }^{17}$ Actually, models with microeconomic rigidities (both upward and downward) can generate strong macroeconomic asymmetries - as Triddon (1987) shows in the context of pricing and Bertola and Caballero (1990) in a more general context- however these are the result of the cross section evolution, not of the microeconomic asymmetry.

"See Samuelson (1955).
} 


\section{A Asymmetric Aggregate Shocks}

Let

$$
\Delta L_{i l}^{*}= \begin{cases}1 & \text { with probability } \lambda_{g} \\ -1 & \text { with probability } 1-\lambda_{g} .\end{cases}
$$

if the aggregate has a good realization $\left(v_{g}\right)$, and

$$
\Delta L_{i t}^{*}= \begin{cases}1 & \text { with probability } \lambda_{b} \\ -1 & \text { with probability } 1-\lambda_{b} .\end{cases}
$$

if the realization of the aggregate is bad $\left(-v_{b}\right)$.

The aggregate shock is "good" with probability $q$ and "bad" with probability $1-q$. The no-drift condition then implies:

$$
v_{b}=-\frac{q}{1-q} v_{g},
$$

and therefore the standard deviation of the aggregate shock, $\sigma_{\Delta A}$, is equal to:

$$
\sigma_{\Delta A}=v_{g} \sqrt{\frac{q}{1-q}}
$$

Denoting the relative contribution of aggregate uncertainty to the uncertainty faced by individual firms by $\delta \equiv \sigma_{\Delta A} / \sigma_{\Delta L^{\bullet}}$, and using the fact that:

$$
\mathrm{E}\left[\Delta L_{i}^{*} \mid v_{g}\right]=v_{g}
$$

and

$$
E\left[\Delta L_{i}^{*} \mid-v_{b}\right]=-\frac{q}{1-q} v_{g},
$$

yields the expressions used in the paper:

$$
\lambda_{g}=\frac{1}{2}\left(1+\delta \sqrt{\frac{1-q}{q}}\right)
$$

and

$$
\lambda_{b}=\frac{1}{2}\left(1-\delta \sqrt{\frac{q}{1-q}}\right) .
$$




\section{B Index of Reallocation Intensity}

The expected change in $L_{i}^{*}$ given a positive aggregate shock is equal to $\left(2 \lambda_{g}-1\right)$, and the conditional variance is:

$$
\sigma_{\Delta L \cdot g}^{2}=\frac{1}{4} \lambda_{g}\left(1-\lambda_{g}\right)
$$

Similar steps show that the expected change in $L_{i}^{*}$ given a negative aggregate shock is equal to $\left(2 \lambda_{b}-1\right)$, and the conditional variance is equal to:

$$
\sigma_{\Delta L \cdot \mid b}^{2}=\frac{1}{4} \lambda_{b}\left(1-\lambda_{b}\right) .
$$

The index $I$ follows trivially from the expressions for the conditional variances.

\section{Identification and VAR's}

Not only microeconomic features may be erased by the natural forces shaping the cross section distributions, but also econometric identification may become more complex. For example, in the job destruction / job creation literature a natural identifying assumption for aggregate versus reallocation "intensity" shocks is whether job creation and destruction move in the same (reallocation shock) or the opposite (aggregate shock) direction. The tables below show, however, that the issue may be more complex once the dynamic behavior of the cross section and the microeconomic strategy are taken into account.

Table 3 reports the fraction of the total number of changes in job creation and job destruction that corresponds to changes of equal sign in the two flow variables (i.e. the type of changes that are typically imputed to reallocation intensity). These fraction are obtained from Monte Carlo simulations with 1,000 replications of samples of 200 observations each. Row 1 corresponds to the case in which hiring and firing are asymmetric at the firm level, as described above, and row 2 corresponds to the case in which hiring and firing at the firm level are symmetric. $\bar{x}_{i}$ represents the average fraction of positively correlated changes (measured every $i$ periods), and $x_{\text {imax }}$ is the maximum fraction of positively correlated changes observed in the 1,000 replications. The first two columns correspond to the asymmetric aggregate case, i.e. when $q=0.75, \lambda_{g}=0.65$ and $\lambda_{b}=0.15$. The following columns correspond to the symmetric aggregate case, i.e. when $q=0.50, \lambda_{g}=0.65=1-\lambda_{b}$. 
Table 3: Aggregate versus Reallocation Shocks

\begin{tabular}{||l||c|c||c|c||}
\hline \hline & $\bar{x}_{1}$ & $x_{1 \max }$ & $\bar{x}_{1}$ & $x_{1 \max }$ \\
\hline \hline Asymm. & $\begin{array}{c}0.11 \\
(0.08)\end{array}$ & 0.33 & $\begin{array}{c}0.05 \\
(0.05)\end{array}$ & $\begin{array}{l}0.25 \\
\end{array}$ \\
\hline Symm. & $\begin{array}{c}0.00 \\
(0.00)\end{array}$ & 0.00 & $\begin{array}{c}0.00 \\
(0.00)\end{array}$ & 0.00 \\
\hline \hline
\end{tabular}

The table shows that when the microeconomic rule is asymmetric the measured average importance of reallocation shocks is 0.11 with a maximum importance of 0.33 . The first surprising result is seen when the microeconomic rule is symmetric; in this case, if no time aggregation problem is present, the measured relative importance of intensity reallocation shocks is identically equal to zero (remember that the presence of reallocation intensity shocks depends on the aggregate asymmetry). Conversely, when the aggregate is symmetric (i.e. there are no intensity reallocation shocks), the measured average importance of these shocks is 0.05 with a maximum of 0.25 . The chief conclusion from this table is that identification of these shocks may be more difficult than expected; microeconomics and aggregation are mixed in complex ways. Table 4 shows results similar to those in Table 3 but for the model with deterministic cycles; $\bar{x}_{i}$ is the fraction of positively correlated changes, with $n_{g}=i$ and $n_{b}=i / 3$, except for the symmetric aggregate row, where $n_{b}=i$. Two results are worth stressing; first, the measured importance of intensity reallocation shocks is increasing on the persistence of the state of nature, and second, in the deterministic framework symmetry at either the micro or the macro level yield zero relative importance for intensity reallocation shocks. 
Table 4: Aggregate versus Reallocation Slocks

\begin{tabular}{||l||c|c|c|c||}
\hline \hline & $\bar{x}_{9}$ & $\bar{x}_{27}$ & $\bar{x}_{54}$ & $\bar{x}_{108}$ \\
\hline \hline Asymm. & 0.42 & 0.45 & 0.60 & 0.73 \\
\hline Symm. & 0.00 & 0.00 & 0.00 & 0.00 \\
\hline Sym aggr. & 0.00 & 0.00 & 0.00 & 0.00 \\
\hline \multicolumn{5}{|c}{$\lambda_{p}=0.65}$. \\
\hline \multicolumn{5}{|c|}{}
\end{tabular}

\section{References}

[1] Bertola, G. and R.J. Caballero, "Kinked Adjustment Costs and Aggregate Dynamics", in NBER Macroeconomic Annual 1990 eds. OJ.Blanchard and S.Fischer, (forthcoming) 1990.

[2] Billingsley, P., Probability and Measure, 1986, $2^{\text {nd }}$ Ed. John Wiley, New York.

[3] Blanchard; O.J. and P.Diamond, "The Beveridge Curve," Brookings Paper on Economic Activity 1989:1, 1989, pp.1-60.

[4] Caballero, R.J. and E.M.R.A. Engel, "The S-s Economy: Aggregation, Speed of Convergence and Monetary Policy Effectiveness," Columbia Working Paper, 1989a.

[5] Caballero, R.J. and E.M.R.A. Engel, "Heterogeneity and Output Fluctuations in a Dynamic Menu Cost Economy," Columbia Working Paper, $1989 \mathrm{~b}$.

[6] Caballero, R.J. and E.M.R.A. Engel, "Aggregation, Coordination and Convergence," Columbia Working Paper, 1990.

[7] Cagan, Ph., Persistent Inflation: Historical and Policy Essays, New York: Columbia, 1979.

[8] Caplin, A. and D. Spulber, "Menu Costs and the Neutrality of Money", Quarterly Journal of Economics 102-4, 1987, pp.703-726.

[9] Cariton, D.W., "The Rigidity of Prices," American Economic Review 76-4, 1986, pp.637658.

[10] Davis, S.J. and J.Haltiwanger, "Gross Job Creation, Gross Job Destruction, and Employment Reallocation," Working paper, 1989. 
[11] Kuran, T., "Asymmetric Price Rigidity and Inflationary Bias," American Economic Review 73-3, 1983, pp.373-382.

[12] Samuelson, P. A., Economics: An Introductory Analysis, McGraw-Hill Inc., 1955.

[13] Tsiddon, D., "On The Stubbornness of Sticky Prices," Hebrew University Working Paper \#174, 1988. 
Figure 1

Stotionory Croas Section

$H=1 F=6$

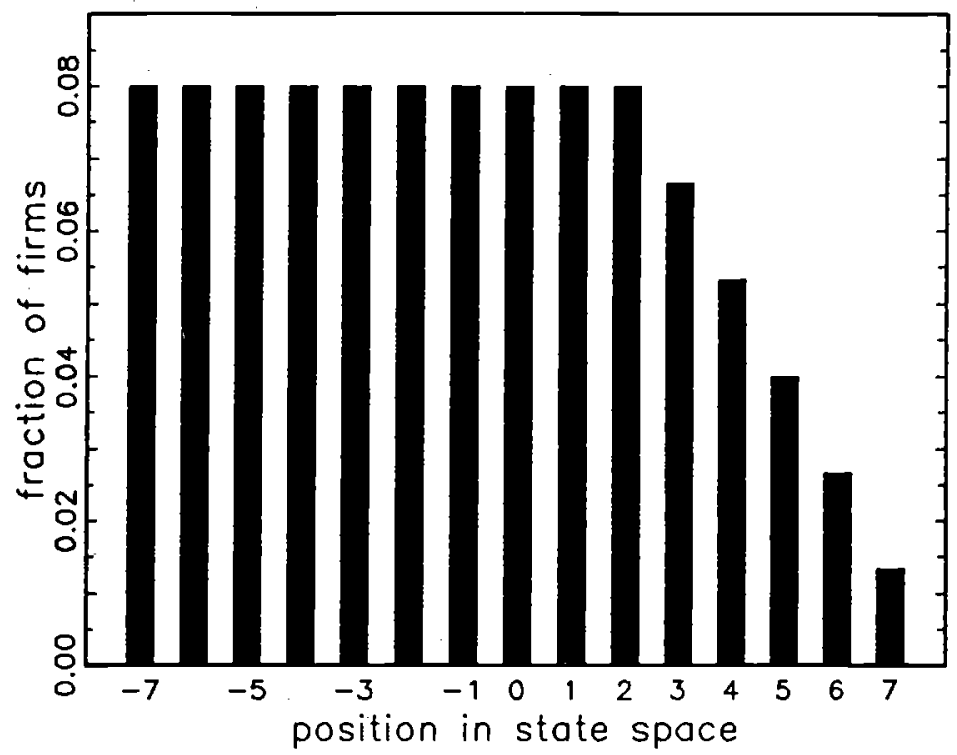




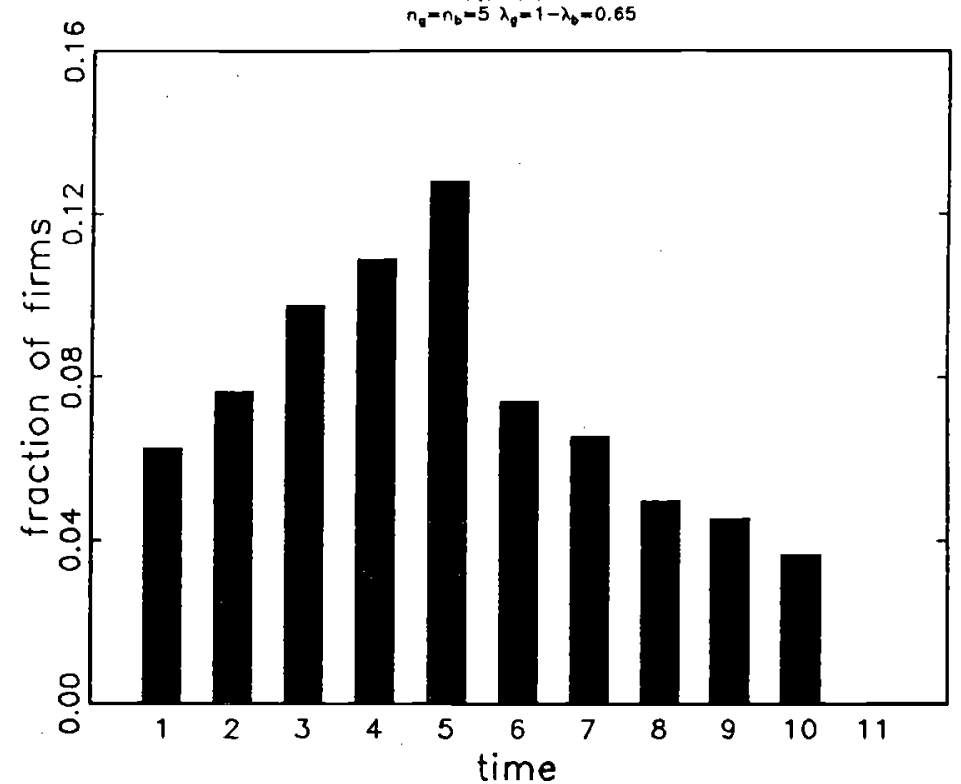

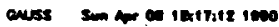

Figure 2b

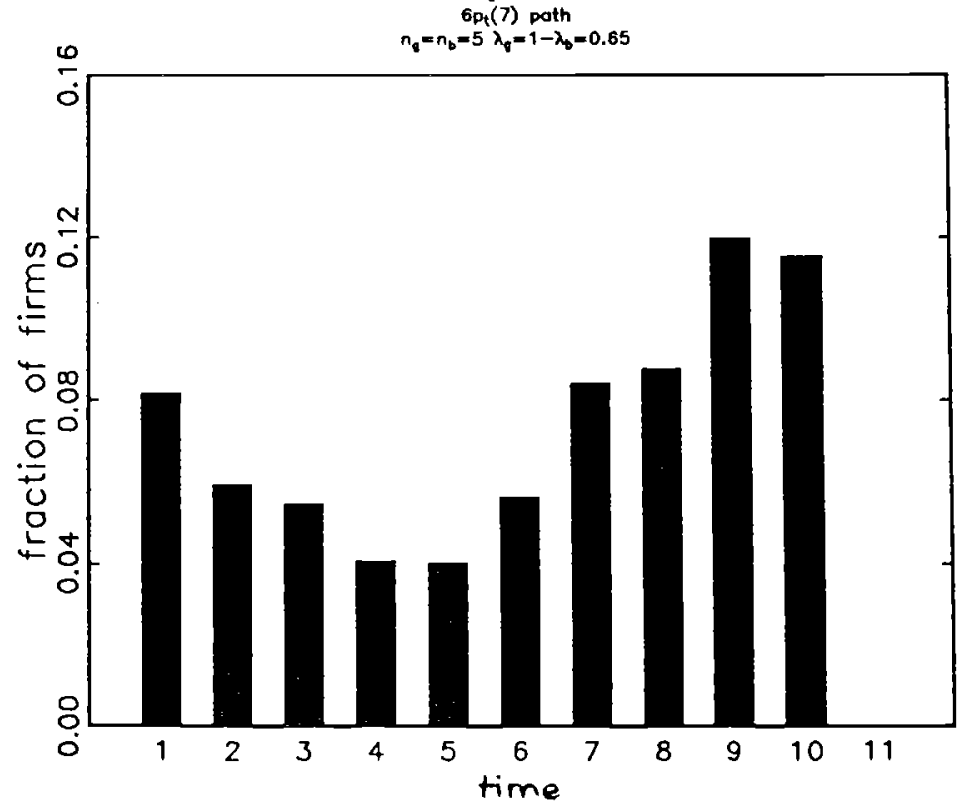


Figure 3o

Aggregate Jab Crootion and Destruction

$n_{0}=n_{0}=5$

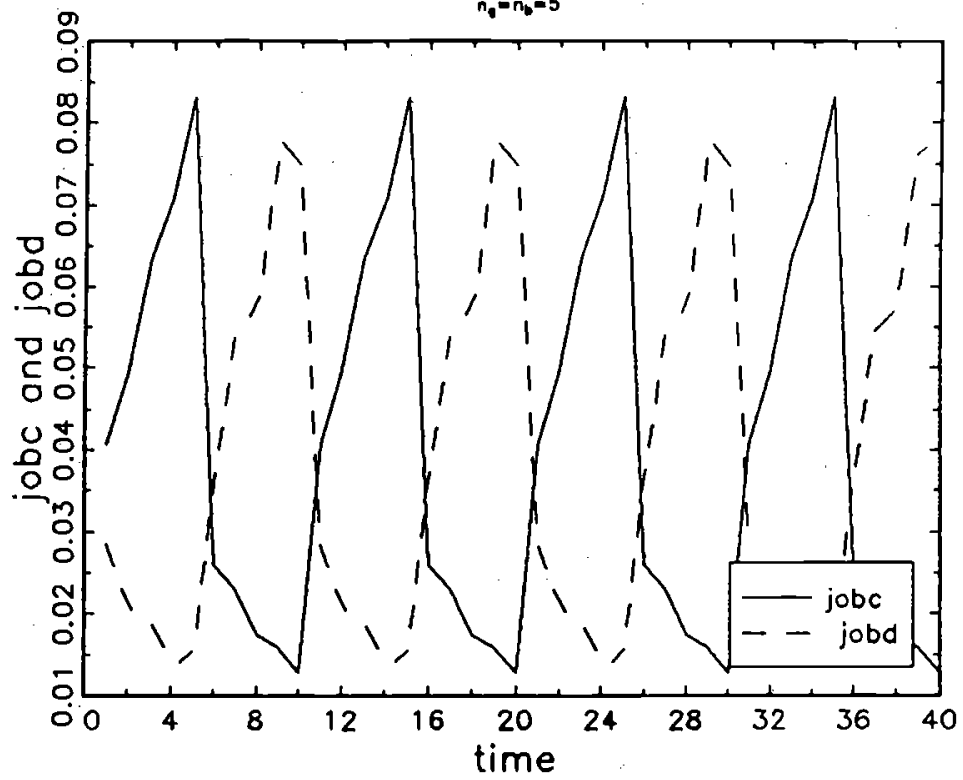

Flgure $3 b$

Aggregate Job Creotion and Destruction

$n_{0}=n_{b}=\mathbf{3 0}$

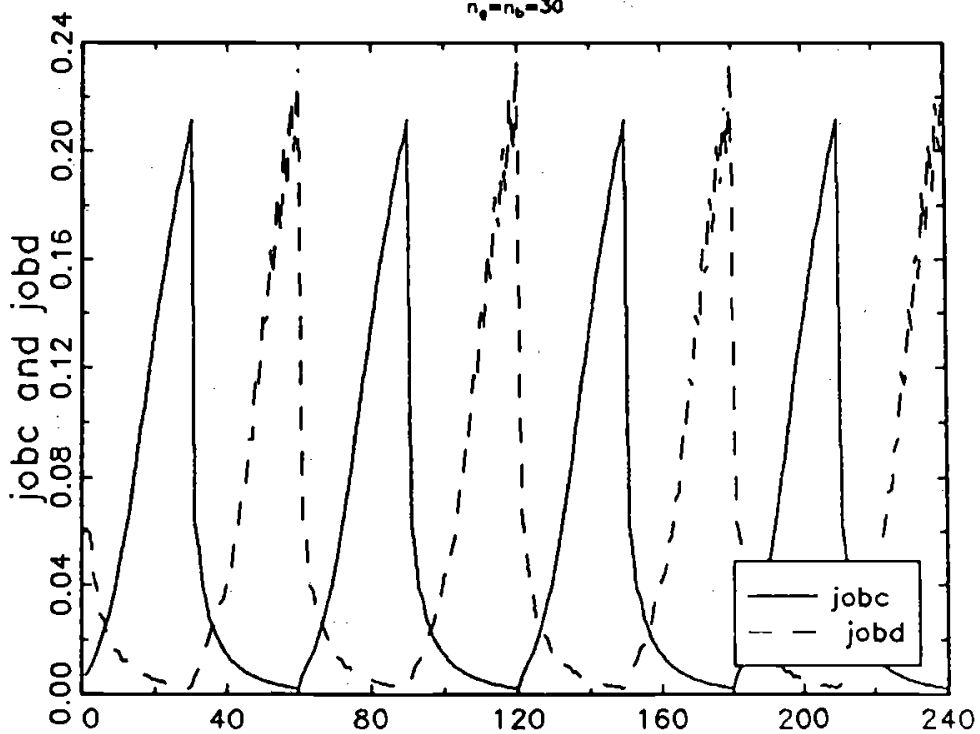


Mus Apr 12 20:50:10 1000

Figure 40

$\ln \left(\sigma_{\text {jobe }}\right)-\ln \left(\sigma_{\text {jobd }}\right)$

osym, $Q=0.5$

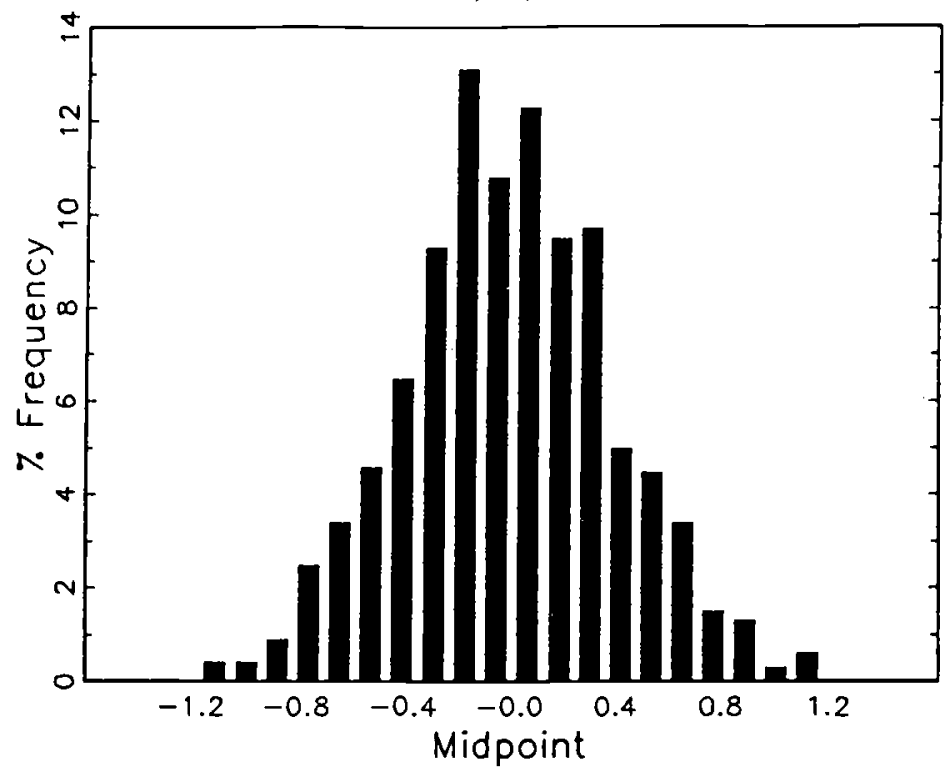

const

$n$ w A 12 210028 160

Figure 4b

$\ln \left(\sigma_{j o b c}\right)-\ln \left(\sigma_{\text {iobd }}\right)$

sym. $q=0.5$

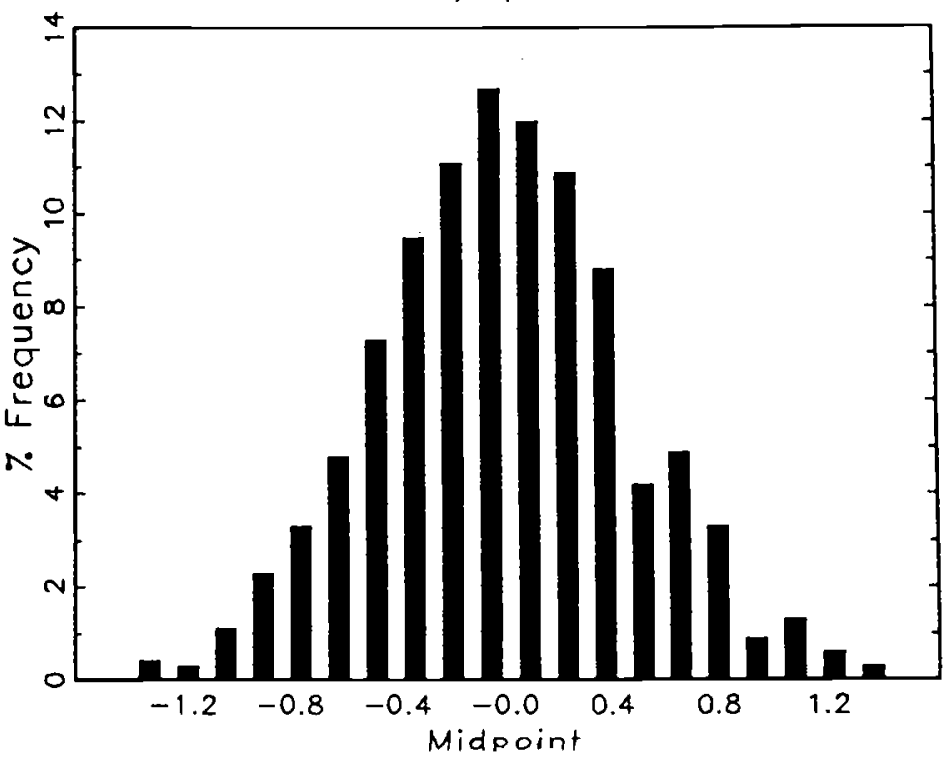


Fiqure 50

$\ln \left(\sigma_{\text {lobc }}\right)-\ln \left(\sigma_{\text {(Obdd }}\right)$

osym. $g=0.75$
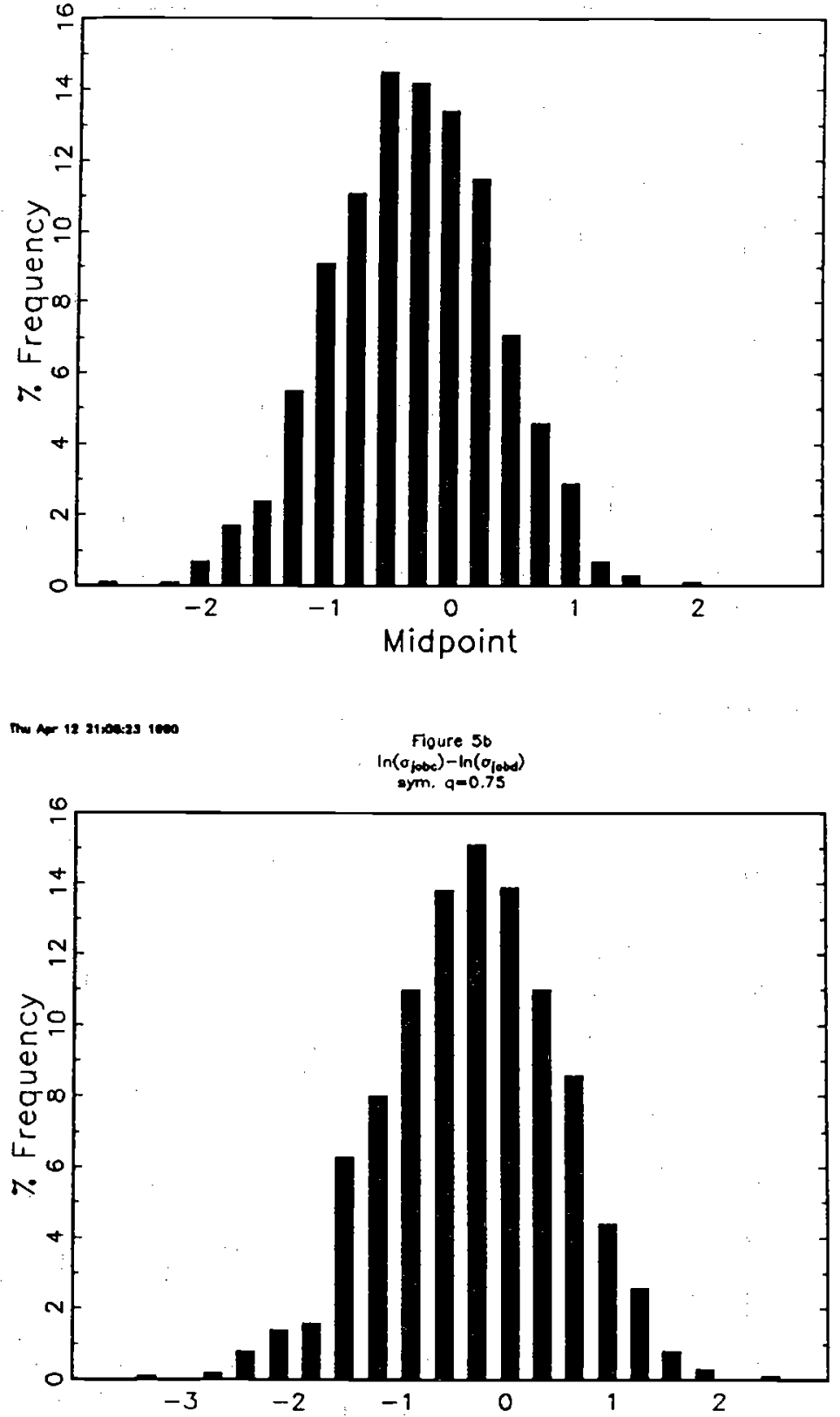
Figure 60

Job Creotion ons Destruction

$n_{0}=12 \quad n_{0}=4$
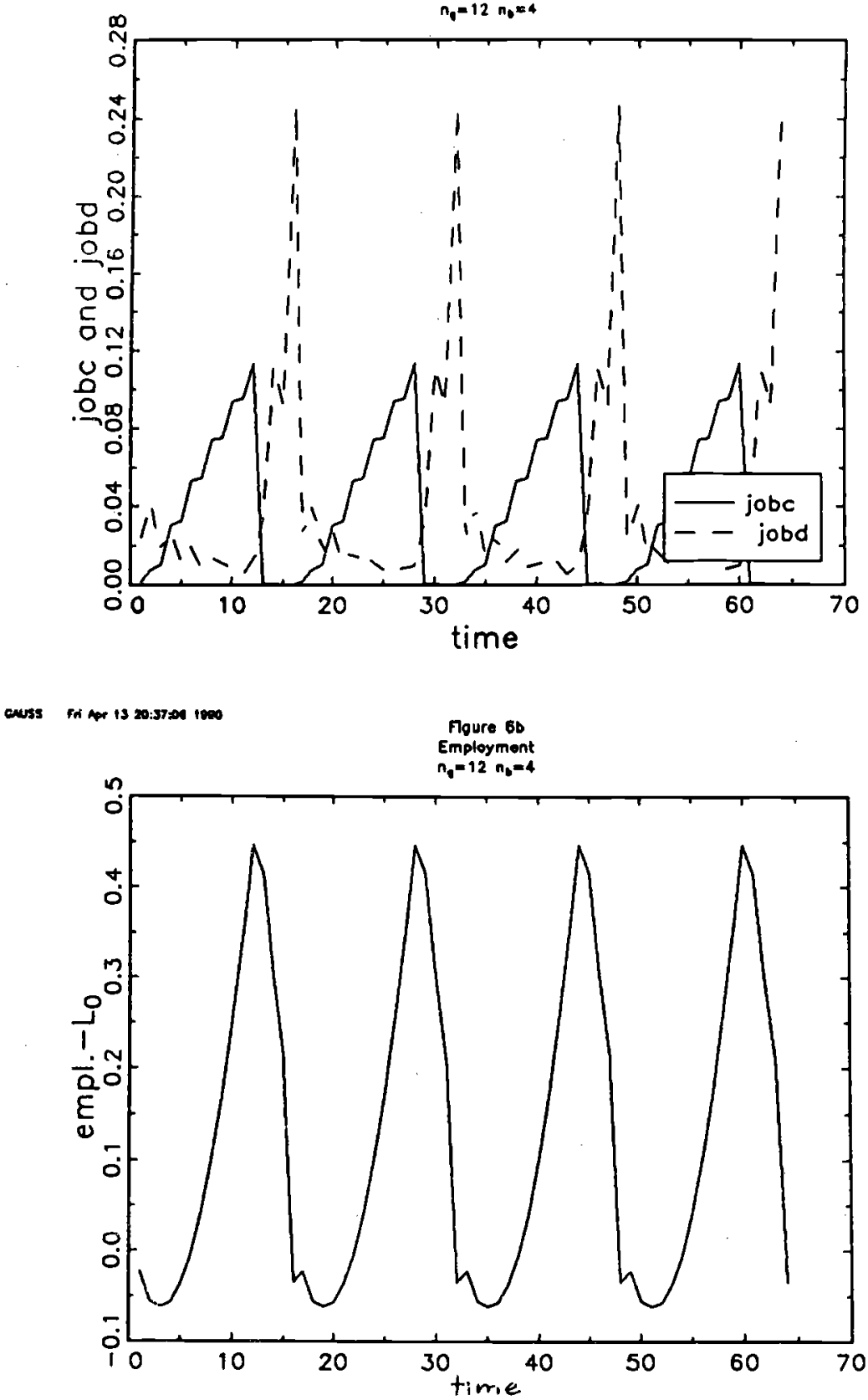